Vipin Kumar*, Shubham Swapnil and V.R. Singh

\title{
Adaptive Algorithm for Solving the Load Flow Problem in Distribution System
}

DOI 10.1515/jisys-2016-0084

Received June 23, 2016; previously published online February 3, 2017.

\begin{abstract}
This paper presents a fast and efficient method for load flow analysis of radial distribution networks. Here, an adaptive algorithm is proposed to analyze the load flow problem of distribution systems. An adaptive algorithm is the combination of backward/forward (BW/FW) sweep and cuckoo search (CS) algorithms. In the proposed method, the optimum load flow analysis of the radial distribution system is attained, while optimizing the voltage and current computation of the BW/FW sweep algorithm. Now, by the CS, the output voltage of the BW/FW sweep algorithm is compared with the standard voltage and optimized. From the optimized voltage and current, load flow parameters like power loss and real and reactive power flow are assessed. The proposed method is implemented using the MATLAB platform and tested into the IEEE 33 bus radial distribution system. The effectiveness of the proposed technique is determined by comparing with the BW/FW algorithm and genetic algorithm-based BW/FW algorithm.
\end{abstract}

Keywords: Voltage deviation index, power loss, cuckoo search algorithm, BW/FW sweep algorithm, genetic algorithm.

\section{Introduction}

In the power system, the distribution system holds a major position because it is the most important point of connection between bulk power and consumers. It engages different kinds of loads, which differ with time, as customer power requirements are not steady [7]. To make certain the electrical power transfer from generators to consumers through the grid system, load flow studies are employed [14]. Regardless of advances in forecasting methods, by a range of uncertainties, load differences continue to be characterized in order to handle these load uncertainties in the load flow problem [3]. The load flow problem is a main problem in the planning, designing, and operating of distribution power systems [16]. At the proposal stage, load flow analysis is employed to verify whether the voltage profiles are anticipated to be within limits throughout the network [15]. In the process stage, the different arrangements for maintaining the necessary voltage profile and minimizing system losses are explored $[4,18]$.

Load flow calculations offer power flows and voltages to adjust the capability of generators, condensers, and tap changers [14] in the distribution system. On the other hand, distribution networks usually have some particular features, such as insufficient information of loads, being radial with sometimes a weakly meshed topology, or high resistance to reactance $\mathrm{r} / \mathrm{x}$ ratios. While performing load flow analysis, those features need to be taken into account [26,32]. As a result, load flow analysis turns out to be a very important part of any power system, for maintaining the network and regulating it inside particular limits becomes just a blind control of some wires in which current flows [6]. Numerous load flow algorithms are particularly designed for distribution systems, and can be separated into two categories. The initial category is based on the general topology of a distribution system and employs bus voltages as state variables to work out the

*Corresponding author: Vipin Kumar, MD University, Rohtak, Haryana, India, e-mail: vipinkumar0780@gmail.com Shubham Swapnil: School of Electrical Engineering, VIT Vellore, Tamil Nadu, India

V.R. Singh: PDM College of Engineering, Bhadurgarh, Haryana, India 
load flow problem. The next category is based on the particular network structures of distribution systems [29]. The procedures and formulations can be accurate or fairly accurate, with values adjusted or unadjusted, meant for either online or offline application, and proposed for either single-case or multiple-case applications $[2,13]$.

There are a number of load flow methods like direct distribution load flow, Gauss implicit Z-bus, Raphson, Gauss Seidal and fast decoupled load flow methods, ladder network theory, backward/forward (BW/FW) sweep distribution load flow, and vector-based distribution load flow, which require extraordinary attention while laboring for weakly mesh connected distribution systems, as in metropolitan cities [20, 22]. In addition, these methods are incompetent in working out such networks [17]. A compensation-based method is used for weakly meshed distribution networks. By a two-step procedure, the radial parts are worked out in which the branch currents are first computed (backward sweep), and next, the bus voltages are revised (forward sweep) [25]. One of the main drawbacks of compensation-based methods is that besides the conventional bus-branch-oriented data format, novel databases have to be built and sustained for these methods [27]. Newly, soft computing methods and optimization algorithms are applied to work out load flow problem such as neural network, fuzzy logic controller, genetic algorithm (GA), particle swarm optimization, etc. [1, 24]. An adaptive algorithm is suggested to examine the load flow problem in this article. The detailed explanation of the suggested technique is offered in Section 3. Previous to that, the current research works are offered in Section 2. The experimental results and discussion are specified in Section 4. At last, Section 5 finishes the article.

\section{Related Works}

A number of research works exist in the literature that are based on the load flow problem of distribution systems. A few of them are reassessed here.

A fuzzy approach load flow for balanced and unbalanced radial distribution systems with incorporation of a load model has been offered by Kalesar and Seifi [9]. The fuzzy set theory suggested a suitable way to model imprecisely expressed information and data that have indecision. A mixture of fuzzy models and techniques for electrical distribution systems is offered in this article. The theories connecting to the fuzzy numbers are simplified into a form apt for practical application. The exploitation of the fuzzy approach is shown by a radial load flow method where the voltage in the substation and the power demands are symbolized by fuzzy numbers.

A competent method for radial load flow solution has been offered by Singh and Ghose [23]. They employed a matrix transformation technique that directly works out the purpose of branch flows in a radial distribution network; thus, it makes the FW/BW sweep-based load flow method more successful and quicker. Their matrix transformation technique uses the radial feature of distribution networks and simple matrix manipulations to attain the needed transformation. The benefit of their method could be realized in any study of radial distribution system where FW/BW sweep-based method has a scope of use.

Gomez-Gonzalez et al. [5] employed the shuffled frog-leaping algorithm and three-phase probabilistic load flow combined with the Monte Carlo method to work out the load flow problem. The main function applied was the power loss expected value. Satisfactory solutions were achieved in a few iterations. Results verify that their suggested method can be used for keeping voltages within needed boundaries at all load nodes of a photovoltaic grid-connected system. To assess the presentation of the photovoltaic system, their suggested method proposed a probabilistic model that takes into account the arbitrary nature of solar irradiance and load. Arithmetical applications were offered and considered regarding the unstable distribution system at IEEE 13 nodes and together with photovoltaic generators at numerous nodes.

For working out the load flow problem in distribution systems, Li et al. [11] proposed the node-branch incidence matrix. The correlation among the branch powers and the node-injection powers were proposed with the node-branch incidence matrix. Next, based on two precise formulas to estimate the voltage drop 
and angle difference, a load flow algorithm for weakly meshed distribution systems was offered. The algorithm decreases the computational complexity and has higher competence by applying active and reactive power rather than complex currents as flow variables. In addition, in order to handle the PV nodes, a solution was brought in based on Thevenin's equivalent circuit. To revise the reactive power injections of PV nodes and to fix the voltage magnitude of PV nodes at particular values, the solution construed a precise calculation formula. Their suggested load flow algorithm fundamentally still belongs to the loopanalysis-based method and has a strong capacity to handle meshed networks. It decreases the iteration number and has a faster calculation speed even when the network turns out to be more meshed and has more PV nodes.

An adapted direct approach for the FW/BW sweep power flow method has been offered by Lisboa et al. [12]. Taking benefit of the particular topological features of the radial network, an algorithm with linear storage complexity was described. The characteristics were summarized in the incidence matrix, which turns out to be a lower triangular matrix after vertex ordering. Their formulation permits working out linear systems of equations as an alternative to explicitly inverting matrices during the iterative process, leading to a lower computational burden. As a result, their suggested method was time and memory efficient. The effects demonstrate that their suggested method develops storage and time complexity without any loss of precision, making it a vigorous and competent method.

\section{Problem Formulation}

In the distribution system, the node voltage, current, power loss, and real and reactive powers are the important factors for analyzing the load flow problem. The load flow analysis of distribution systems is dissimilar from other power system networks. The equality and inequality constraints are described for maintaining the optimal load flow of the distribution system. Then, the real and reactive power flow equation can be used for reducing the power losses of the system [8]. The traditional radial distributions load flow analysis does not succeed in assembling the necessities in both performance and vigor features in the distribution system applications. The BW/FW sweep method works out the variance node voltage and current of the network. It influences the load flow parameters of the suggested system, i.e. power loss and real and reactive power flow. Therefore, voltage and current optimization should be preferred for examining the load flow of the radial distribution system. The objective function required for the optimization process is described in Eq. (1).

\section{(a) Objective function}

The objective function of the proposed method is given by Eq. (1):

$$
\begin{gathered}
O_{b}=\operatorname{Min}\left(f_{1}, f_{2}\right), \\
f_{1}=V_{D I}=1-\max \left(1-\frac{V_{i}}{V_{r}}\right), \\
f_{2}=P_{L}=\left(\left|I_{i}^{b c}\right|^{2} R_{i}\right)_{i=1}^{N_{b}},
\end{gathered}
$$

where $V_{D I}$ and $P_{L}$ are the voltage deviation index and power loss, respectively. From Eq. (2), the voltage deviation is evaluated from the maximized voltage profile. In this process, the maximized voltage profile value is evaluated initially [10]. After that, the voltage deviation of the system is obtained. In the above equations, $I_{b}$ and $R_{i}$ are the bus current and resistance, respectively. The objective function is described based on the constraints, which are defined as follows. 


\section{(b) Constraints}

The node voltage is specified within the limits. The limits are set by using the following equation:

$$
V_{i}^{\min } \leq V_{i} \leq V_{i}^{\max },
$$

where $V_{i}$ is the node voltage of the $i^{\text {th }}$ bus specified with the limits $(0.9-1.06 \mathrm{pu})$. The specified objective function is focused to the power flow constraints. The power flow equations given specify the real and reactive power flow among the buses. It can be explained by the subsequent equations:

$$
\begin{aligned}
& P_{i}=\left|V_{i}\right| \sum_{j=1}^{N_{b}}\left|Y_{i j}\right|\left|V_{j}\right| \cos \left(\alpha_{i j}+\delta_{j}-\delta_{i}\right) \\
& Q_{i}=\left|V_{i}\right| \sum_{j=1}^{N_{b}}\left|Y_{i j}\right|\left|V_{j}\right| \sin \left(\alpha_{i j}+\delta_{j}-\delta_{i}\right) .
\end{aligned}
$$

In the above equations, $P_{i}$ and $Q_{i}$ are the active and reactive power of the $i^{\text {th }}$ bus, $N_{b}$ is the number of buses, $Y_{i j}$ is the bus admittance matrix, $V_{i}$ and $V_{j}$ are voltages of the bus, $\alpha_{i j}$ is the angle of $Y_{i j}$, and $\delta_{i}$ and $\delta_{j}$ are voltage angles of bus $i$ and $j$. The above-stated constraints are optimally determined by using the suggested objective function. The suggested adaptive algorithm can optimize the system parameters and distribute the optimum load flow of the radial distribution system. In the subsequent section, the suggested technique is briefly described.

\subsection{Adaptive Algorithm for Analyzing the Load Flow Problem in the Distribution System}

The load flow problem of the distribution system is analyzed by using the proposed method. Here, the BW/ FW sweep algorithm-based cuckoo search (CS) algorithm is proposed. During the iteration process, the BW/ FW algorithm has variance voltage and current computation problem. The voltage and current at each node should be optimized in order to develop the load flow analysis of the radial distribution network. These activities can meet the optimum load flow of the necessary distribution system. The fundamental data that are necessary in the suggested algorithm are the active and reactive powers of each node, categorization for sending and receiving nodes, and the positive sequence impedance model for the entire branches. The input data of this suggested algorithm is specified by node branch-oriented data employed by most utilities. In this paper, the CS algorithm is used to reduce the voltage deviation index and power loss of the distribution

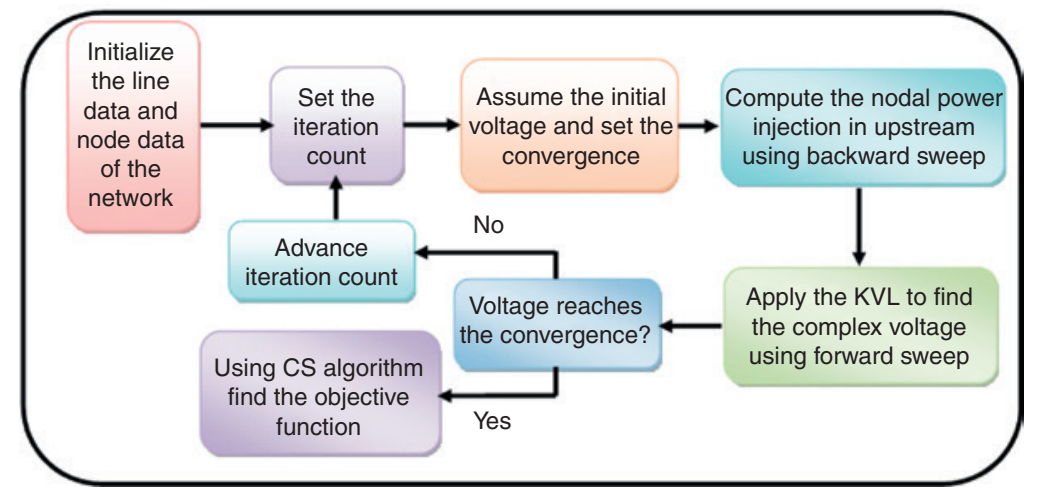

Figure 1: Load Flow Analysis of the Distribution System Using the Proposed Method. 
system, and also to improve the node voltage profile and convergence speed of the BW/FW sweep algorithm. The general block diagram of the proposed method is illustrated in Figure 1. The overall process of the proposed algorithm is explained in the following sections.

\subsubsection{BW/FW Sweep Algorithm}

This algorithm encloses two stages of the process, which are the backward and forward processes. It is a very important method. The steps of backward and forward processes of this algorithm are elucidated below.

\subsubsection{Backward Process}

\section{(i) Initialization}

Here, the bus and line data of the distribution system are initialized and read.

(ii) Evaluation

In the evaluation process, the load current $i_{l}$ and the node currents $i_{n}$ are computed by means of the nodal injected power $S_{i}$ and its voltage vector $V_{i}$. In every iteration $i$, branch currents are combined from the loads to origin, which are specified in the following:

$$
I_{b c}^{i}=\left(-\Delta_{m}\right) I_{n}^{i},
$$

where $I_{b c}{ }^{i}$ is the branch current, $I_{n}^{i}$ is the node current, and $\Delta_{m}$ is the upper triangular matrix.

\subsubsection{Forward Process}

In this process, the node voltage $\left(V_{n}\right)$ is updated from the origin to load according to Kirchhoff's voltage law. This can be attained by employing the prior branch current vector $I_{b c}{ }^{i}$ and branch impedance $Z$. The voltage can be explained in Eq. (5).

$$
V^{i+1}=V_{0}-X
$$

where $X=\Delta_{m} B_{Z} I_{b c}{ }^{i}$.

\subsubsection{Convergence Criteria}

Repeat the above procedure until the convergence condition is satisfied. The required convergence range is given in the following equation:

$$
0.001 \leq\left|V_{i}^{i+1}-V_{i}\right| .
$$

The output voltage of the BW/FW sweep algorithm is necessary for the upgrading process because the improvement of the voltage computation and branch current can change the system parameters, i.e. power loss and real and reactive power flow. This can be attained by the CS optimization algorithm, which is momentarily explained in the next section.

\subsubsection{CS Algorithm to Optimize the Voltage and Current for Analyzing the Load Flow}

The CS algorithm is one of the optimization algorithms that are inspired by the breeding behavior of the cuckoos, and is easy to implement. The basic CS optimization algorithm is primarily based on the natural obligate brood parasitic behavior of some cuckoo species in combination with the Levy flight behavior of some birds and fruit flies. Cuckoos are naturally fascinating birds because of the lovely sound they produce and because of their intelligent reproduction strategy. Cuckoos lay their eggs in the nest of other host birds; 
they also tend to destroy the eggs of other birds to increase the hatching probability of their own egg so that when the eggs are hatched, their chicks are fed by the other birds. Some species of cuckoo mimic the nature of host birds so that the host could not recognize them and give the cuckoo a better chance of survival. If the host bird identifies the cuckoo egg despite all efforts of cuckoo, the host may either destroy the cuckoo egg or abandon the nest and build a new nest somewhere else [21].

\section{(i) Searching capabilities of cuckoo}

In the CS algorithm, three idealized rules are used.

(a) Each cuckoo lays one egg at a time, and dumps its egg in a randomly chosen nest.

(b) The best nests with high-quality eggs will carry over the next generations.

(c) The number of available host nests is fixed, and the egg laid by a cuckoo is discovered by the host bird with a probability of $P \varepsilon[0,1]$. In this case, the host bird can either throw the egg away or abandon the nest to build a completely new nest in a new location.

In this paper, the CS algorithm is used for minimizing the voltage deviation index and power loss of the system to analyze the load flow problem. The CS algorithm is used to optimize the voltage and current of the BW/FW sweep algorithm. It is used to improve the convergence speed of the BW/FW sweep algorithm. The inputs of the CS algorithm are the node voltage and branch current. The output of the proposed algorithm is the voltage deviation index and the power loss $\left(P_{\text {Loss }}\right)$. The optimized voltage and current can be identified by the minimized fitness function. Then, the resultant voltage and current can deliver the optimum load flow. The detailed description of the algorithm is given below. The flowchart of the CS algorithm is illustrated in Figure 2.

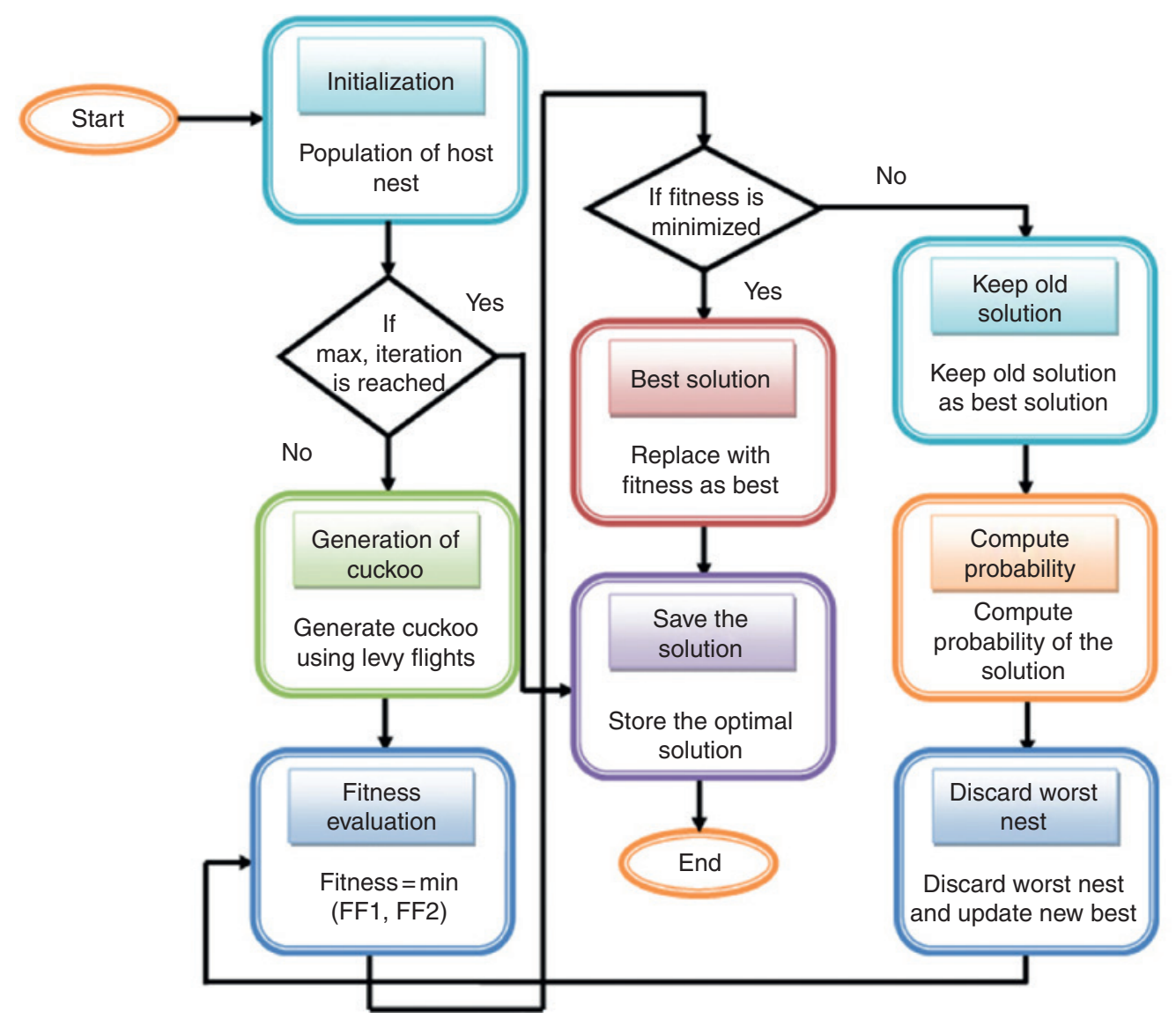

Figure 2: Flowchart of the CS Algorithm. 


\section{(ii) Steps for CS algorithm}

\section{(a) Initialization}

In this section, the host nests are randomly initiated. Here, nest is an array of size $n$.

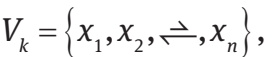

$$
\begin{aligned}
& I_{k}=\left\{y_{1}, y_{2}, \vec{\sim}, y_{n}\right\} \text {, }
\end{aligned}
$$

where $V_{k}$ and $I_{k}$ are the node voltage and branch current, respectively. In the proposed algorithm, the voltage and current profile of the every node is given as the input, i.e. output voltage and current of the BW/FW sweep algorithm. Here, voltage and current are randomly generated within the voltage and current profile limits of the BW/FW sweep algorithm. Then, each nest $x_{n}$ is a solution vector to the optimization problem. It can hold $n$ number of variables, which are optimized so as to minimize the objective function.

\section{(b) Generating new cuckoo}

A cuckoo randomly generates new solutions by using the Levy function and determines the quality of solutions. The Levy function $[\operatorname{Levy}(\lambda)]$ is represented as follows:

$$
X_{K}^{t+1}=X_{k}^{t}+\alpha \oplus \operatorname{Levy}(\lambda) .
$$

The cuckoo is evaluated using the objective function to determine the quality of the solutions.

\section{(c) Fitness function}

Here, the fitness function $(F F)$ of all the inputs is evaluated by using the following formula:

$$
(F F)=\min \left(f_{1}, f_{2}\right),
$$

where the power loss $\left(P_{\text {loss }}\right)$ depends on the branch current variations, and so it is calculated by Eq. (3). Then, the voltage deviation index is calculated based on Eq. (2).

Thereafter, the quality of the solutions is measured. If the fitness function is minimized, then the current solution is saved as an optimal solution. Otherwise, the previous solution is kept as a best solution.

\section{(d) Discard worst nest}

In this part, the worst nests are discarded based on their probability $p_{a}$ values and new ones are built using Eq. (1). Subsequently, the best solutions are ranked based on their quality. Then, the present best solutions are identified as optimal solutions.

\section{(e) Stopping criterion}

This process is repeated until the termination iteration is reached. From the output of the CS algorithm, the voltage deviation index and power loss are minimized while optimizing the voltage and current. The optimal set of minimum power loss and voltage deviation index of the system is evaluated. Once the process is finished, the required load flow constraints, such as power loss and real and reactive power flow, are calculated by using the optimized voltage and current. The analysis part of the proposed method is briefly explained in the following section.

\section{Results and Discussion}

The proposed method is implemented in MATLAB working platform and tested in the IEEE 33 bus distribution system [19, 28]. Here, the BW/FW sweep algorithm-based CS algorithm is proposed to analyze the load flow of the distribution system. In the proposed method, the voltage and current are optimized for the load flow analysis of the distribution system. From the optimized values, the voltage deviation index and power loss are reduced. The bus and line data of the distribution system are analyzed, which contain 33 nodes and 


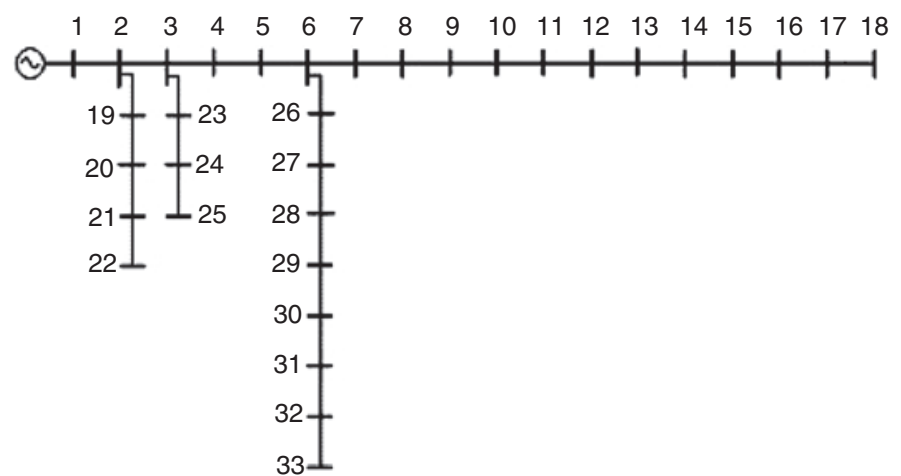

Figure 3: Structure of the IEEE 33 Bus Distribution System.

32 branches. The performance of the proposed method is evaluated. In order to verify the effectiveness and robustness of the proposed method, it is compared with existing techniques such as the BW/FW sweep algorithm and GA-based BW/FW sweep algorithm.
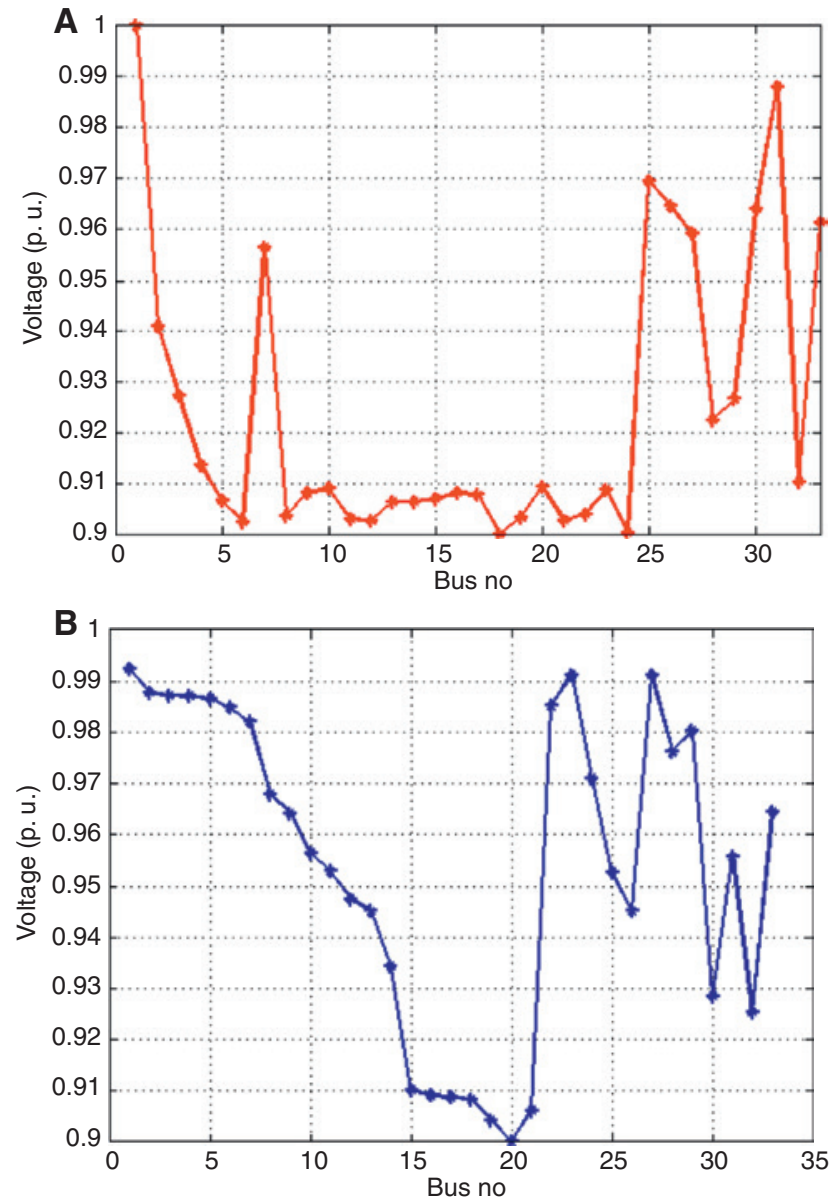

Figure 4: (A) Performance Analysis of Voltage Profile Using the BW/FW Sweep Method. (B) Performance Analysis of Voltage Profile Using the Proposed Method. 

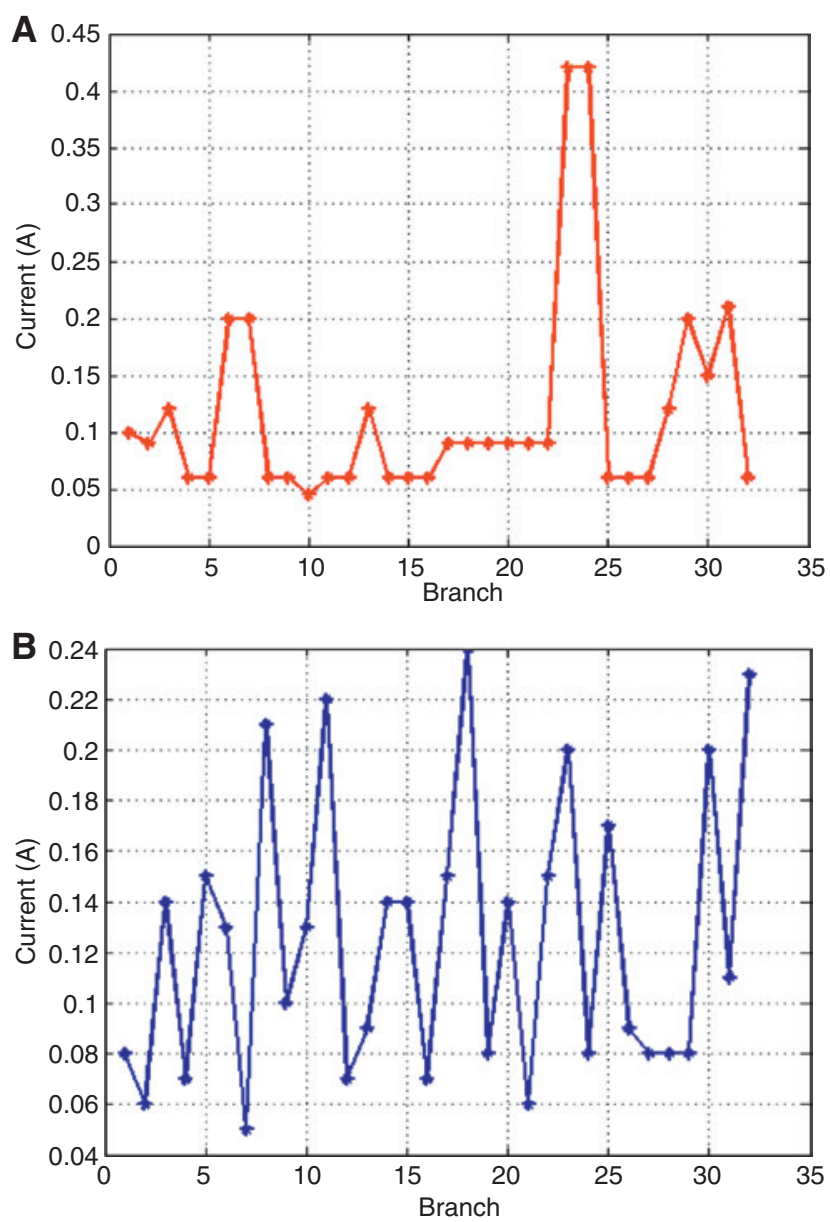

Figure 5: (A) Performance Analysis of the Current Profile Using the B/F Sweep Method. (B) Performance Analysis of the Current Profile Using the Proposed Method.

\subsection{Performance Analysis of the Proposed and Existing Techniques}

In this section, the performance of the proposed method is analyzed in terms of the voltage, current, and real and reactive power of the IEEE 33 bus distribution system. The voltage and current profiles are determined and depicted in Figures 3 and 4. Also, the performances of power loss and voltage deviation index are analyzed. The tested distribution system structure is shown in Figure 5 [30, 31].

Tables 1 and 2 represent the analysis of the voltage and real and reactive power flow of the distribution system by using various methods. The performances of voltage and current profiles are evaluated using the BW/FW sweep, and the proposed method are illustrated in Figures 4A, B and 5A, B.

Figures 4A, B and 5A, B furnish the load flow analysis performance of the proposed method. Initially, the IEEE 33 bus distribution system load flow analysis using the BW/FW sweep algorithm is obtained. The corresponding irregular mismatch node voltage develops the power loss, and the real and reactive power analysis becomes poor, reflecting the power loss of the testing radial distribution system as about 0.32092 pu. These problems can be resolved by the proposed adaptive BW/FW sweep algorithm. In the proposed method, the voltage and current are optimized by using the CS algorithm, thereby reducing the power loss and voltage deviation index values. The proposed method optimizes the better node voltage and the branch current using the CS algorithm. The optimized voltage and current performances are compared with the BW/ FW sweep method, GA, and proposed method, which are illustrated in Figures 6 and 7. Parameters such as 
Table 1: Node Voltages of the Distribution System by Using the Proposed and Existing Methods.

\begin{tabular}{|c|c|c|c|}
\hline \multirow[t]{2}{*}{ Node no. } & \multicolumn{3}{|r|}{ Node voltage (pu) } \\
\hline & Bw/Fw Sweep algorithm & GA-Based BW/FW sweep algorithm & Proposed method \\
\hline 1 & 1.0000 & 1.0000 & 1.0000 \\
\hline 2 & 0.9410 & 0.9873 & 0.9877 \\
\hline 3 & 0.9274 & 0.9866 & 0.9872 \\
\hline 4 & 0.9138 & 0.9853 & 0.9871 \\
\hline 5 & 0.9068 & 0.9671 & 0.9866 \\
\hline 6 & 0.9026 & 0.9557 & 0.9848 \\
\hline 7 & 0.9565 & 0.9540 & 0.9820 \\
\hline 8 & 0.9010 & 0.9471 & 0.9680 \\
\hline 9 & 0.9061 & 0.9403 & 0.9642 \\
\hline 10 & 0.9065 & 0.9336 & 0.9565 \\
\hline 11 & 0.9068 & 0.9266 & 0.9528 \\
\hline 12 & 0.9050 & 0.9258 & 0.9475 \\
\hline 13 & 0.9055 & 0.9222 & 0.9451 \\
\hline 14 & 0.9002 & 0.9223 & 0.9343 \\
\hline 15 & 0.9061 & 0.9019 & 0.9102 \\
\hline 16 & 0.9087 & 0.9067 & 0.9091 \\
\hline 17 & 0.9029 & 0.9054 & 0.9088 \\
\hline 18 & 0.9084 & 0.9017 & 0.9083 \\
\hline 19 & 0.9099 & 0.8992 & 0.9043 \\
\hline 20 & 0.9014 & 0.9637 & 0.9000 \\
\hline 21 & 0.9076 & 0.8994 & 0.9061 \\
\hline 22 & 0.9039 & 0.9360 & 0.9851 \\
\hline 23 & 0.9050 & 0.8972 & 0.9912 \\
\hline 24 & 0.9074 & 0.9671 & 0.9710 \\
\hline 25 & 0.9864 & 0.9516 & 0.9527 \\
\hline 26 & 0.9473 & 0.9178 & 0.9451 \\
\hline 27 & 0.9247 & 0.9911 & 0.9913 \\
\hline 28 & 0.9671 & 0.9725 & 0.9761 \\
\hline 29 & 0.9396 & 0.9754 & 0.9804 \\
\hline 30 & 0.9765 & 0.9204 & 0.9285 \\
\hline 31 & 0.9815 & 0.9365 & 0.9556 \\
\hline 32 & 0.9054 & 0.9112 & 0.9253 \\
\hline 33 & 0.9031 & 0.9564 & 0.9645 \\
\hline
\end{tabular}

the accurate node voltage, radial distribution system power loss, and real and reactive power are effectively analyzed is shown in Table 3.

The line loss of the system is analyzed by using the proposed and the existing methods, which are furnished in Table 4 and Figure 8. Here, the proposed method highly reduces the line loss of the system when compared with other techniques, which is illustrated in Figure 9. The performance deviations are analyzed in terms of the voltage deviation index and the power loss by using various methods.

Here, the performances of the voltage deviation index are determined by using the proposed and existing methods. By using the proposed method, the voltage deviations are calculated and the power loss of the system is also evaluated. Then, the deviation voltage values are compared with those of the BW/FW sweep algorithm and GA-based BW/FW sweep algorithm. Here, the proposed method voltage deviation index is 0.0072 . In the BW/FW sweep algorithm and the GA-based BW/FW sweep algorithm, the voltage deviation indices are 0.0626 and $0.0208 \mathrm{pu}$, respectively. By using the proposed technique, the losses are determined. The performances are compared with the BW/FW sweep technique and GA-based BW/FW sweep technique. In the proposed method, the normal losses are reduced up to $0.0052 \mathrm{pu}$. Furthermore, the power losses are reduced up to 
Table 2: Analysis of Active and Reactive Power.

\begin{tabular}{|c|c|c|c|c|c|c|c|}
\hline \multicolumn{2}{|c|}{ Node no. } & \multicolumn{2}{|c|}{ B/F Sweep algorithm } & \multicolumn{2}{|c|}{ GA-Based BW/FW sweep algorithm } & \multicolumn{2}{|r|}{ Proposed method } \\
\hline $\begin{array}{l}\text { From } \\
\text { node }\end{array}$ & $\begin{array}{r}\text { To } \\
\text { node }\end{array}$ & $\begin{array}{r}\text { Active power } \\
(\mathrm{pu})\end{array}$ & $\begin{array}{r}\text { Reactive power } \\
(\mathrm{pu})\end{array}$ & $\begin{array}{r}\text { Active power } \\
(\mathrm{pu})\end{array}$ & $\begin{array}{r}\text { Reactive power } \\
(\mathrm{pu})\end{array}$ & $\begin{array}{r}\text { Active power } \\
(\mathrm{pu})\end{array}$ & $\begin{array}{r}\text { Reactive power } \\
\text { (pu) }\end{array}$ \\
\hline 1 & 2 & 0.7839 & -1.2209 & 1.3731 & 0.6358 & 0.8179 & -1.2739 \\
\hline 2 & 3 & 0.7819 & 1.7085 & 1.4210 & 2.0270 & 0.8788 & 1.9202 \\
\hline 3 & 4 & 2.4420 & -0.3481 & 1.4040 & 2.1866 & 2.8812 & -0.4107 \\
\hline 4 & 5 & 2.0943 & -0.9697 & 2.4720 & -0.7194 & 2.5513 & -1.1813 \\
\hline 5 & 6 & 0.5617 & -0.6503 & 0.8173 & 0.5299 & 0.6502 & -0.7528 \\
\hline 6 & 7 & 0.4868 & -1.6455 & 0.9642 & 1.5017 & 0.5257 & -1.7771 \\
\hline 7 & 8 & 0.7847 & 1.2220 & 0.2235 & -1.5200 & 0.9162 & 1.4270 \\
\hline 8 & 9 & 0.6555 & 0.2965 & 0.2335 & 0.7894 & 0.8016 & 0.3626 \\
\hline 9 & 10 & 0.4550 & 0.5268 & 0.7454 & 0.3371 & 0.5342 & 0.6186 \\
\hline 10 & 11 & 2.5213 & 3.9268 & 4.5489 & 2.9493 & 3.1014 & 4.8302 \\
\hline 11 & 12 & 1.3041 & 2.0311 & 2.8862 & -0.4114 & 1.6587 & 2.5832 \\
\hline 12 & 13 & 0.3373 & 0.2939 & 0.2916 & 0.4541 & 0.4492 & 0.3914 \\
\hline 13 & 14 & 0.5060 & -0.7881 & 0.9608 & -0.4448 & 0.6647 & -1.0352 \\
\hline 14 & 15 & 0.3020 & -1.0210 & 0.7110 & 1.1074 & 0.3903 & --1.3193 \\
\hline 15 & 16 & 0.4919 & 0.7660 & 0.1678 & -1.2155 & 0.6766 & 1.0537 \\
\hline 16 & 17 & 0.3905 & 0.0557 & 0.4694 & -0.2123 & 0.5503 & 0.0784 \\
\hline 17 & 18 & 0.2523 & -0.8815 & 0.4886 & 1.0677 & 0.3532 & -1.2340 \\
\hline 20 & 19 & 2.7867 & -2.4284 & 4.8155 & 0.6864 & 3.7837 & -3.2973 \\
\hline 19 & 20 & 0.1163 & -0.3932 & 0.4255 & -0.2759 & 0.1633 & -0.5521 \\
\hline 20 & 21 & 0.0058 & 1.3137 & 1.5492 & -1.6072 & 0.0081 & 1.8324 \\
\hline 21 & 22 & 0.7152 & 0.1020 & 0.8313 & -0.2419 & 0.9615 & 0.1371 \\
\hline 22 & 23 & 1.0293 & -1.1918 & 0.5320 & 1.7983 & 1.3472 & -1.5598 \\
\hline 23 & 24 & 0.1070 & 0.7278 & 0.7924 & 0.3584 & 0.1426 & 0.9697 \\
\hline 24 & 25 & 0.7183 & -0.8316 & 0.2403 & -0.8122 & 0.6791 & -0.7863 \\
\hline 25 & 26 & 8.2569 & 2.4028 & 3.4221 & 2.9822 & 5.2735 & 1.5346 \\
\hline 26 & 27 & 0.9131 & -6.2090 & 1.3756 & 3.0057 & 0.5616 & -3.8185 \\
\hline 27 & 28 & 1.3121 & -0.6075 & 0.3949 & -0.6150 & 0.7442 & -0.3446 \\
\hline 28 & 29 & 1.8478 & -1.1749 & 0.9067 & -0.4101 & 0.9471 & -0.6022 \\
\hline 29 & 30 & 4.5116 & -2.0407 & 0.7410 & 1.6191 & 1.9393 & -0.8772 \\
\hline 30 & 31 & 0.0103 & -2.3213 & 0.0032 & -0.7225 & 0.0036 & -0.8234 \\
\hline 31 & 32 & 4.2275 & 6.5840 & 1.7231 & 1.5016 & 1.3527 & 2.1067 \\
\hline 32 & 33 & 4.2169 & 4.8824 & 1.5886 & 0.4623 & 1.2059 & 1.3962 \\
\hline
\end{tabular}

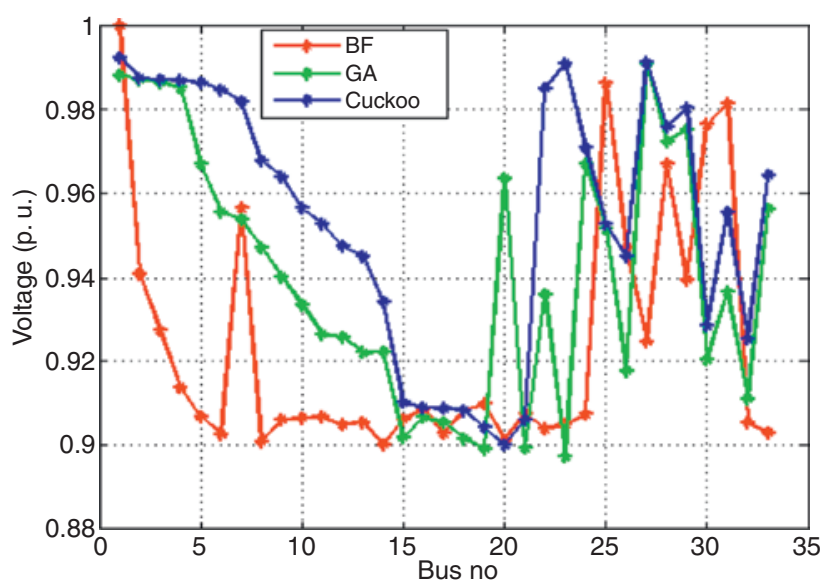

Figure 6: Voltage Comparison between the Proposed Method and the Existing Method. 


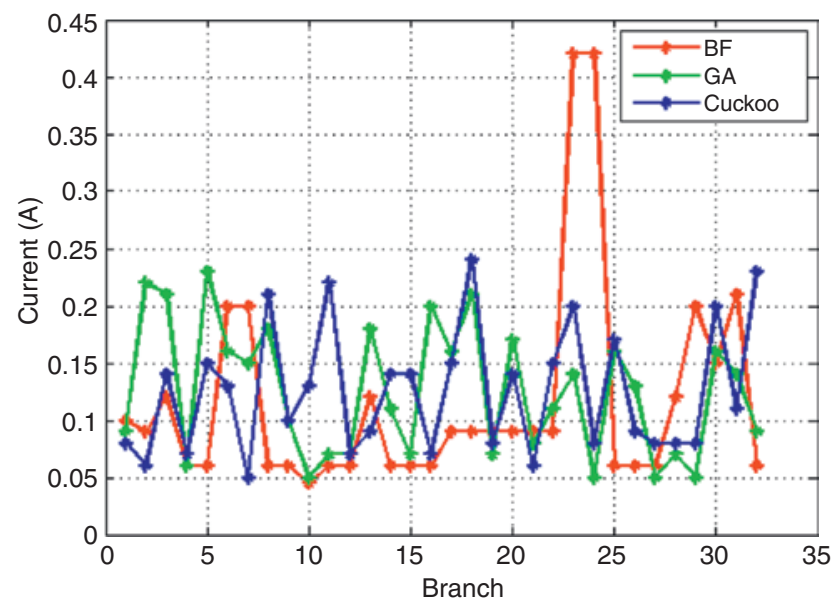

Figure 7: Current Profile Comparison between the Proposed and Existing Methods.

Table 3: Power Losses of the Distribution System.

Branch No.

Power loss

BW/FW Sweep algorithm

GA-Based BW/FW sweep algorithm

Proposed method

\begin{tabular}{|c|c|c|c|}
\hline 1 & 0.0062 & 0.0062 & 0.0062 \\
\hline 2 & 0.0025 & 0.0024 & 0.0111 \\
\hline 3 & 0.0033 & 0.0030 & 0.0028 \\
\hline 4 & 0.0009 & 0.0112 & 0.0115 \\
\hline 5 & 0.0018 & 0.0009 & 0.0007 \\
\hline 6 & 0.0047 & 0.0047 & 0.0038 \\
\hline 7 & 0.0178 & 0.0151 & 0.0036 \\
\hline 8 & 0.0023 & 0.0023 & 0.0023 \\
\hline 9 & 0.0023 & 0.0019 & 0.0005 \\
\hline 10 & 0.0077 & 0.0062 & 0.0002 \\
\hline 11 & 0.0008 & 0.0008 & 0.0008 \\
\hline 12 & 0.0033 & 0.0144 & 0.0132 \\
\hline 13 & 0.0049 & 0.0049 & 0.0041 \\
\hline 14 & 0.0013 & 0.0123 & 0.0003 \\
\hline 15 & 0.0017 & 0.0025 & 0.0015 \\
\hline 16 & 0.0029 & 0.0023 & 0.0136 \\
\hline 17 & 0.0037 & 0.0032 & 0.0028 \\
\hline 18 & 0.0008 & 0.0006 & 0.0004 \\
\hline 19 & 0.0076 & 0.0054 & 0.0034 \\
\hline 20 & 0.0021 & 0.0035 & 0.0017 \\
\hline 21 & 0.0036 & 0.0030 & 0.0028 \\
\hline 22 & 0.0023 & 0.0023 & 0.0010 \\
\hline 23 & 0.0989 & 0.0456 & 0.0020 \\
\hline 24 & 0.0987 & 0.0563 & 0.0027 \\
\hline 25 & 0.0084 & 0.0073 & 0.0052 \\
\hline 26 & 0.0011 & 0.0011 & 0.0006 \\
\hline 27 & 0.0024 & 0.0032 & 0.0054 \\
\hline 28 & 0.0098 & 0.0075 & 0.0072 \\
\hline 29 & 0.0127 & 0.0042 & 0.0020 \\
\hline 30 & 0.0137 & 0.0115 & 0.0103 \\
\hline 31 & 0.0085 & 0.0068 & 0.0028 \\
\hline 32 & 0.0008 & 0.0012 & 0.0002 \\
\hline
\end{tabular}


Table 4: Power Loss and Voltage Deviation Index.

\begin{tabular}{lrrrrrr}
\hline Power loss (pu) & & & & \multicolumn{2}{c}{ Voltage deviation index (pu) } \\
\cline { 6 - 8 } $\begin{array}{l}\text { B/F sweep } \\
\text { method }\end{array}$ & $\begin{array}{r}\text { GA-Based BW/FW } \\
\text { sweep method }\end{array}$ & $\begin{array}{r}\text { Proposed } \\
\text { method }\end{array}$ & & $\begin{array}{r}\text { B/F sweep } \\
\text { method }\end{array}$ & $\begin{array}{r}\text { GA-Based BW/FW } \\
\text { sweep method }\end{array}$ & $\begin{array}{r}\text { Proposed } \\
\text { method }\end{array}$ \\
\cline { 6 - 8 } 0.0084 & 0.0073 & 0.0052 & & 0.0626 & 0.0208 & 0.0072 \\
\hline
\end{tabular}
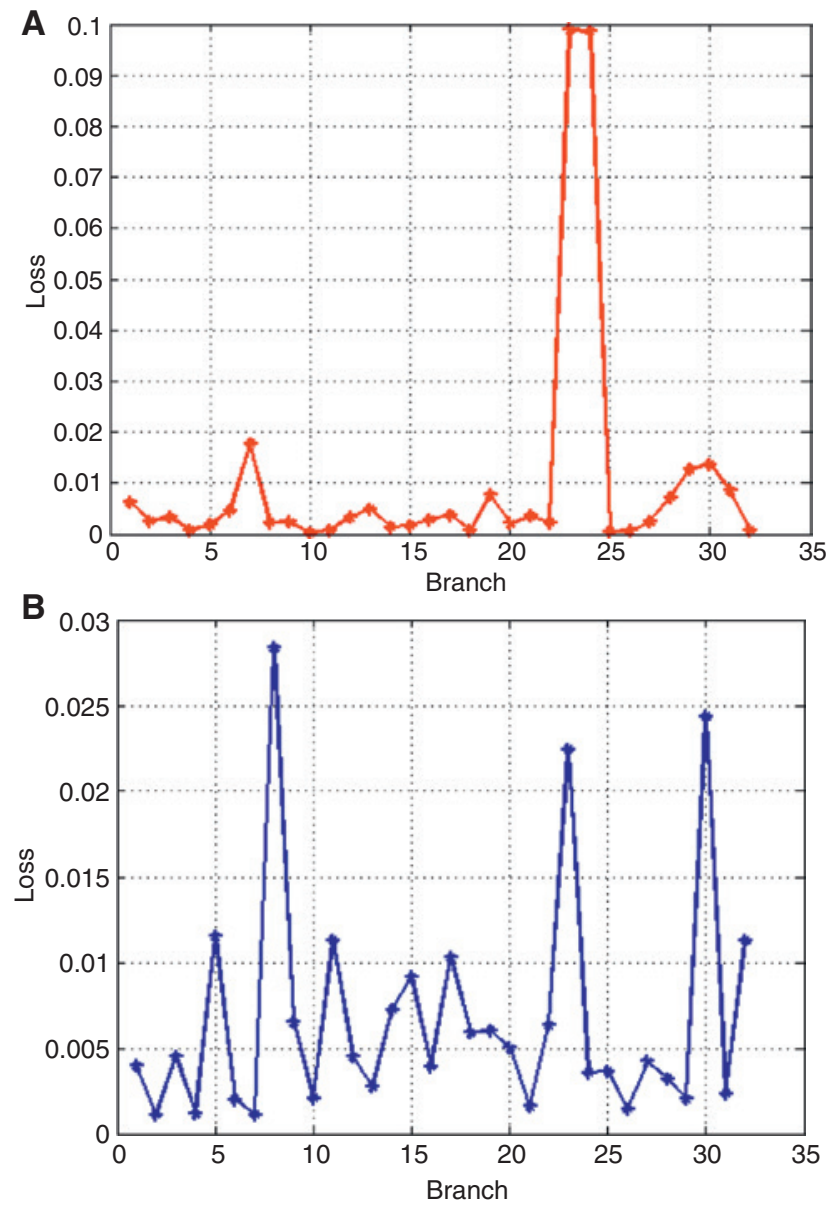

Figure 8: (A) Line Loss Using the B/F Sweep Method. (B) Line Loss Using the Proposed Method.

0.0073 and $0.0084 \mathrm{pu}$ in the GA-based BW/FW sweep technique and the BW/FW sweep approach, respectively. When compared to other techniques, the loss reduction is high in the proposed method. Therefore, the proposed hybrid method achieves better outputs compared with the other techniques.

\section{Conclusion}

The proposed method was implemented in MATLAB platform. The BW/FW algorithm-based CS algorithm was used to solve the load flow problem of the distribution system. The voltage deviation index and the power losses were reduced using the proposed method. The voltage deviation index was based on the deviation voltage. The proposed technique was tested with the IEEE 33 bus system. From the optimized voltage, load 


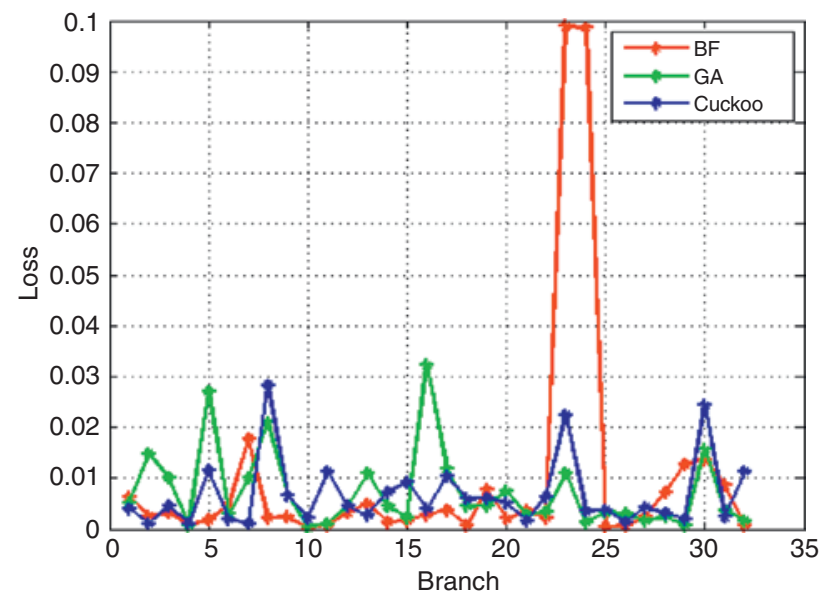

Figure 9: Line Loss Comparison between the Proposed Method and Existing Method.

flow parameters like power loss and real and reactive power flow were evaluated. The total power loss and voltage deviations were analyzed, and the corresponding results were discussed. The deviation voltage was analyzed with the BW/FW sweep method and GA-based BW/FW sweep technique, which proved that the proposed method achieved better outputs. From the comparison results, it was shown that the proposed method is a sound and effective load flow technique for the radial distribution system, which is competent compared with other techniques.

\section{Bibliography}

[1] P. Acharjee and K. Ali, Load flow analysis using decoupled fuzzy load flow under critical conditions, Int. J. Eng. Sci. Technol. 3 (2011), 111-118.

[2] A. Arunagiri and B. Venkatesh, Harmonic load flow for radial distribution systems, J. Eng. Sci. Technol. 6 (2011), 300-310.

[3] W. C. Briceno Vicente, R. Caire and N. Hadjsaid, Probabilistic load flow for voltage assessment in radial systems with wind power, Elect. Power Energy Syst. 41 (2012), 27-33.

[4] J. Chakavorty and M. Gupta, A new method of load flow solution of radial distribution networks, Int. J. Elect. Commun. Eng. 5 (2012), 9-22.

[5] M. Gomez-Gonzalez, F. J. Ruiz-Rodriguez and F. Jurado, A binary SFLA for probabilistic three-phase load flow in unbalanced distribution systems with technical constraints, Elect. Power Energ. Syst. 48 (2013), 48-57.

[6] A. González, F. M. Echavarren, L. Rouco and T. Gómez, A sensitivities computation method for reconfiguration of radial networks, IEEE Trans. Power Syst. 27 (2012), 1294-1301.

[7] T. E. Grebe, Application of distribution system capacitor banks and their impact on power quality, IEEE Trans. Ind. Appl. 32 (1996), 714-719.

[8] S. K. Injeti, V. K. Thunuguntla and M. Shareef, Optimal allocation of capacitor banks in radial distribution systems for minimization of real power loss and maximization of network savings using bio-inspired optimization algorithms, Elect. Power Energ. Syst. 69 (2015), 441-455.

[9] B. M. Kalesar and A. R. Seifi, Fuzzy load flow in balanced and unbalanced radial distribution systems incorporating composite load model, Elect. Power Energ. Syst. 32 (2010), 17-23.

[10] I. Kumaraswamy, S. Tarakalyani and B. V. Prasanth, Optimal location of distributed generation on radial distribution system for loss reduction and voltage profile improvement, Afr. J. Comput. ICT. 7 (2014).

[11] H. Li, A. Zhang, X. Shen and J. Xu, A load flow method for weakly meshed distribution networks using powers as flow variables, Elect. Power Energ. Syst. 58 (2014), 291-299.

[12] A. C. Lisboa, L. S. M. Guedes, D. A. G. Vieira and R. R. Saldanha, A fast power flow method for radial networks with linear storage and no matrix inversions, Elect. Power Energ. Syst. 63 (2014), 901-907.

[13] S. F. Mekhamer, S. A. Soliman, M. A. Moustafa and M. E. El-Hawary, Load flow solution of radial distribution feeders: a new contribution, Elect. Power Energy Syst. 24 (2002), 701-707. 
[14] K. K. Murthy and S. V. Jaya Ram Kumar, Three phase unbalanced radial distribution load flow method, Int. Refer. J. Eng. Sci. 1 (2012), 39-42.

[15] N. Mwakabuta and A. Sekar, Comparative study of the IEEE 34 node test feeder under practical simplifications, in: Proceedings of North American 39th Symposium on Power, pp. 484-491, 2007.

[16] D. Pawar, C. Radhakrishna, H. S. Jain and S. Ravichander, Non-iterative radial network power flow with parameter uncertainties: interval mathematics, in: Proceedings of IEEE Conference, pp. 1-7, 2009.

[17] K. Prakash and M. Sydulu, An effective topological and primitive impedance based distribution load flow method for radial distribution systems, in: Proceedings of IEEE Third International Conference on DRPT, pp. 1044-1049, 2008.

[18] A. D. Rana, J. B. Darji and M. Pandya, Backward/forward sweep load flow algorithm for radial distribution system, Int. J. Sci. Res. Dev. 2 (2014), 398-400.

[19] R. Ranjan, B. Venkatesh and D. Das, Voltage stability analysis of radial distribution networks, Elect. Power Compon. Syst. 31 (2013), 501-511.

[20] P. U. Reddy, S. Sivanagaraju and P. Sangameswararaju, Power flow analysis of three phase unbalanced radial distribution system, Int. J. Adv. Eng. Technol. 3 (2012), 514-524.

[21] S. Roy and S. S. Chaudhuri, Cuckoo search algorithm using Levy flight: a review, Int. J. Modern Educ. Comput. Sci. 12 (2013), 10-15.

[22] T. Sathiyanarayanan and M. Sydulu, Load flow analysis for radial and mesh connected distribution systems, Int. J. Sci. Res. 3 (2014), 522-528.

[23] S. Singh and T. Ghose, Improved radial load flow method, Elect. Power Energ. Syst. 44 (2013), 721-727.

[24] A. Singh, S. Dixit and L. Srivastava, Particle swarm optimization-artificial neural network for power system load flow, Int. J. Power Syst. Oper. Energy Manage. 1 (2011), 6.

[25] S. Sivanagaraju, J. V. Rao and M. Giridhar, A loop based load flow method for weakly meshed distribution network, ARPN J. Eng. Appl. Sci. 3 (2008), 55-59.

[26] C. Sreenivasulu, G. Madhusudhana Rao and B. V. Sanker Ram, Reliable load flow solution for controlling power network by FACTS devices, Int. J. Emerg. Technol. Adv. Eng. Certif. J. 3 (2013), 739-744.

[27] J. B. V. Subrahmanyam, C. Radhakrishna and K. Pandukumar, A simple and direct approach for unbalanced radial distribution system three phase load flow solution, Res. J. Appl. Sci. Eng. Technol. 2 (2010), 452-459.

[28] S. A. Taher, A. Karimian and M. Hasani, A new method for optimal location and sizing of capacitors in distorted distribution networks using PSO algorithm, Simul. Model. Pract. Theory 19 (2011), 662-672.

[29] J. H. Teng and C. Y. Chang, A novel and fast three-phase load flow for unbalanced radial distribution systems, IEEE Trans. Power Syst. 17 (2002), 1238-1244.

[30] B. Venkatesh and R. Ranjan, Optimal radial distribution system reconfiguration using fuzzy adaptation of evolutionary programming, Elect. Power Energ. Syst. 25 (2003), 775-780.

[31] B. Venkatesh, R. Ranjan and H. B. Gooi, Optimal reconfiguration of radial distribution systems to maximize loadability, IEEE Trans. Power Syst. 19 (2004), 260-266.

[32] X. Yang, M. S. Choi, I. H. Lim and S. J. Lee, Load flow analysis for distribution automation system based on distributed load modeling, J. Elect. Eng. Technol. 2 (2007), 329-334. 